\title{
A ROBUST GOLD-SILICON EUTECTIC WAFER BONDING TECHNOLOGY FOR VACUUM PACKAGING
}

\author{
Yuhai Mei, G. Roientan Lahiji, and Khalil Najafi \\ Center for Wireless Integrated Microsystems \\ The University of Michigan \\ 1301 Beal Ave., Ann Arbor, MI 48109
}

\begin{abstract}
A uniform, high-yield, reproducible, gold-silicon eutectic bonding technology for wafer-level MEMS vacuum packaging has been successfully demonstrated. A device wafer containing a polysilicon layer is eutectically bonded to a silicon cap wafer containing an electroplated gold bond ring. The soft eutectic flows over non-planar surfaces containing insulated feedthroughs of polysilicon $(1.2 \mu \mathrm{m}$-thick,). The two wafers are first baked in vacuum at $300^{\circ} \mathrm{C}$ for 60 minutes, brought into intimate contact under a pressure of $1 \mathrm{MPa}$, and subsequently bonded at $\sim 400^{\circ} \mathrm{C}$ for 30 minutes in a vacuum of $\sim 0.25 \mathrm{mTorr}$. A bond yield of $>95 \%$ is achieved across 4 " wafers, and excellent reproducibility is achieved from wafer to wafer. The lowpressure inside the vacuum-sealed cavity is monitored using a thin $(\sim 2.5 \mu \mathrm{m}$-thick) diaphragm of insulated polysilicon. After bonding and diaphragm release, the diaphragm buckles under the one atmosphere of pressure by $>27 \mu \mathrm{m}$. The buckling in the diaphragms has been maintained for more than 14 weeks. Vacuum sensors are being fabricated to more exactly monitor the pressure inside the package.
\end{abstract}

\section{INTRODUCTION}

Low-cost, simple, and reproducible hermetic/vacuum packaging technologies are required for many microsystems, including resonant devices and RF MEMS. Several groups, including ours, are developing new techniques for implementing small packages [1-5]. Most of these involve bonding of two wafers, a package (cap) silicon/glass wafer, and a device silicon wafer. Several wafer bonding techniques, including adhesive, glass frit, solder, eutectic, silicon fusion/direct and anodic bonding have been used. Of these, eutectic bonding is one of the most attractive because it is easy to use, it forms a soft eutectic to allow bonding over non-planar surfaces, it can be done at slightly above the eutectic temperature $\left(363^{\circ} \mathrm{C}\right)$, and it does not out-gas.

Although Au-Si eutectic has long been used for wafer bonding and packaging [1-3], few have reported its successful use in vacuum packaging. There are several reasons for this, including non-uniform eutectic flow, void formation, insufficient eutectic material in between wafers causing nonuniform bonding, oxidation of bond surfaces, and poor surface contact/adhesion. Furthermore, few published reports have presented data showing full wafer-level bonding [2]. The main problem with $\mathrm{Au}-\mathrm{Si}$ eutectic bonding has been a lack of uniformity over an entire wafer, and lack of reproducibility from wafer to wafer. This paper presents a uniform, high-yield, reproducible, silicon-gold eutectic wafer-level bonding technology used for vacuum encapsulation of MEMS. The paper presents a detailed description of the bonding process and the steps necessary to ensure a uniform and reproducible bond. The vacuum inside packaged cavities is monitored using a thin flexible diaphragm that is fabricated on one side of the packaged cavity. This wafer-level bonding technology can be applied to a variety of MEMS devices which require a prescribed level of vacuum or pressure, excellent bonding strength, low fabrication cost, and high reliability and yield.

\section{GOLD-SILICON EUTECTIC BONDING}

Gold-silicon eutectic formation occurs at $363^{\circ} \mathrm{C}$ for 19 atomic $\% \mathrm{Si}$, as shown in the phase diagram in Fig. 1 . The eutectic can be used to bond two wafers, or be used for hermetic and vacuum packaging. To form the eutectic, silicon and gold have to be provided to the bond interface. Typically, the gold is deposited on one of the wafers to a desired thickness, and the silicon is provided either from the bulk of one of the wafers, or from thin films deposited on one or both wafers. Figure 2, for example, shows two wafers, a device wafer containing feedthroughs and a top poysilicon layer, and a cap wafer containing the package cavity and an electroplated gold bond ring. When the two wafers are brought into contact and heated to above $363^{\circ} \mathrm{C}$, silicon is supplied from both the cap wafer (underneath the gold ring) and the device wafer (the polysilicon layer), and will react with gold to initiate the eutectic liquid formation. Upon cooling, the bond consists of $\mathrm{Au}-\mathrm{Si}$ hypereutectic phase and represents a typical strong diffusional bond [6]. We will present additional detail and discussion on the bonding mechanisms, and different bond interfaces and materials later in the paper.

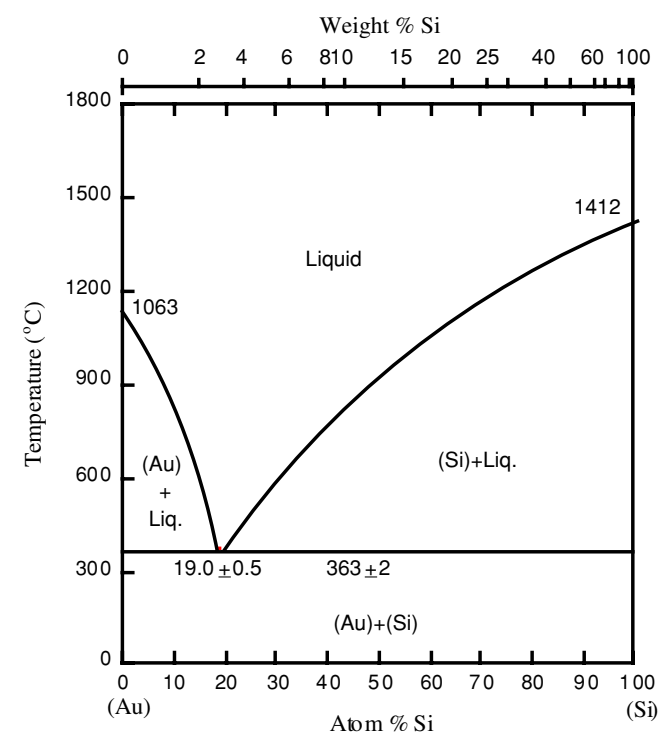

Figure 1. Binary phase diagram of Au-Si alloy. 


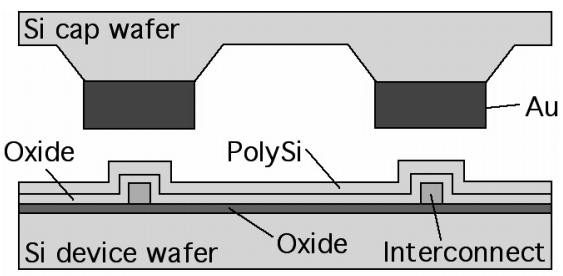

(a)

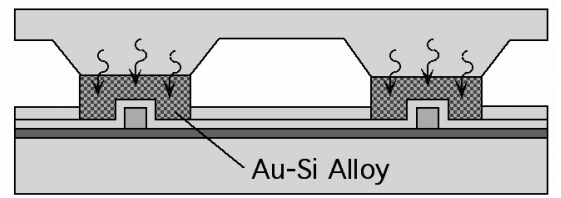

(b)

Figure 2. Schematic diagram of Au-Si eutectic bonding: at (a) room temperature, (b) $>363^{\circ} \mathrm{C}$.

\section{TEST DEVICES AND FABRICATION}

To develop and characterize the bonding process, a set of test wafers and devices were fabricated. The experiments involved two wafers, a device wafer which supports the required bonding layers, and a silicon cap wafer that contains the gold bond ring and the package cavity. Silicon is used as the cap wafer because it can be easily machined, matches the thermal expansion of the device wafer, is low cost, and has excellent mechanical characteristics. In the following, the fabrication process for each of these wafers is first described, and the details of wafer bonding process are then presented.

Figure 3 shows the fabrication process of the cap wafer. Thermal oxide $(0.5 \mu \mathrm{m})$ is first grown on the backside of the cap wafer in order to protect the wafer during the following $\mathrm{KOH}$ wet etching process. Next, the bonding layer is formed on the front surface. It consists of a layer of $\mathrm{Ti}(200 \AA)$, followed by a seed layer of $\mathrm{Au}(1000 \AA)$, and a top layer of $\mathrm{Cr}(500 \AA)$. The chromium is patterned in areas where the thick gold bond ring is to be formed. The bond ring is typically $200 \mu \mathrm{m}$ wide. Gold is now electroplated to a thickness of $4-8 \mu \mathrm{m}$ through a thick photoresist mask. The plating mask and the underlying $\mathrm{Ti} / \mathrm{Au} / \mathrm{Cr}$ layer are now removed and the wafer is etched in $\mathrm{KOH}$ for 60 minutes to form a $\sim 50 \mu \mathrm{m}$ deep recess to create the package cavity. This completes the processing of the cap wafer.

Figure 4 shows the fabrication process of the device wafer. In these initial experiments, the device wafer simply supports a multi-layer of thin dielectric and polysilicon films. These films eventually form a thin diaphragm that can be used to monitor the pressure inside the package. Thermal oxide $(2 \mu \mathrm{m})$ and LPCVD $\mathrm{Si}_{3} \mathrm{~N}_{4}(1800 \AA)$ and $\mathrm{SiO}_{2}(2300 \AA)$ are first deposited on a silicon substrate for electrical insulation followed by the deposition of $1.2 \mu \mathrm{m}$ LPCVD polysilicon. The polysilicon is then phosphorus-doped and patterned to form electrical interconnects and feedthroughs. In order to prevent the diffusion of $\mathrm{Au}$ into this interconnect layer during the bonding process, a LPCVD $\mathrm{SiO}_{2}(2300 \AA) / \mathrm{Si}_{3} \mathrm{~N}_{4}(1800 \AA)$ barrier layer is deposited. Next, a second layer of LPCVD polysilicon $(0.5 \mu \mathrm{m})$ is deposited; this polysilicon film forms the bonding layer on the device wafer. The wafers are now ready to be bonded. Note that the diaphragms are released after the two wafers are bonded together. Diaphragm release is performed by etching the device wafer from the back side in a DRIE as illustrated in Fig. 4(b).

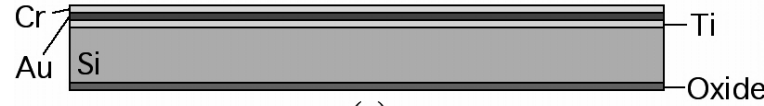

(a)

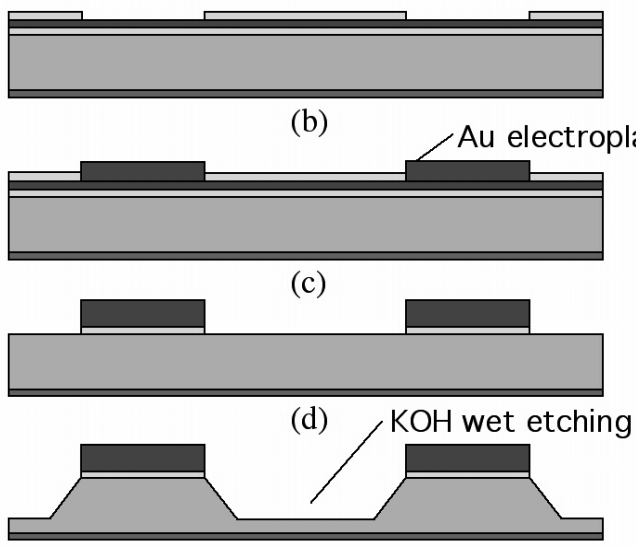

(e)

Figure 3. Fabrication process flow of the silicon cap wafer.

The cap and device wafers are now aligned, and then bonded. The most critical step in the process is the exact bonding sequence. Bonding is performed in an Electronic Vision EV-420 bonder, where the temperature and pressure can be controlled. After placement in the bonding chamber, the chamber is pumped down to a vacuum level of about $0.25 \mathrm{~m}$ Torr. The wafers are then baked at $300^{\circ} \mathrm{C}$ for about 60 minutes to release any residual materials off of the surfaces of the package cavity. Note that this baking is performed at $300^{\circ} \mathrm{C}$, which is far below the eutectic point. The wafers are now brought into intimate contact under an applied pressure of $1 \mathrm{MPa}$, and the wafer stack is heated to the desired bonding temperature. Most of our bonds were performed at a temperature of $400^{\circ} \mathrm{C}$ for about 20-30 minutes. The applied pressure helps distribute the eutectic throughout the bond interface. Now, the wafers are cooled down to room temperature and removed from the bonder. It should be noted that it is desirable to rapidly cool down the wafer stack after bonding in order to obtain a fine microstructure for the gold-silicon alloy after it solidifies.

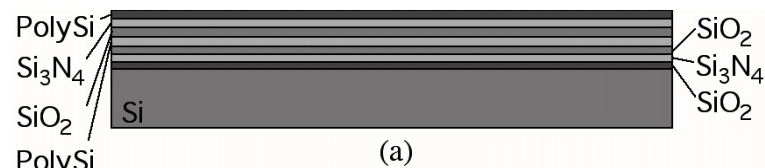

PolySi

(a)

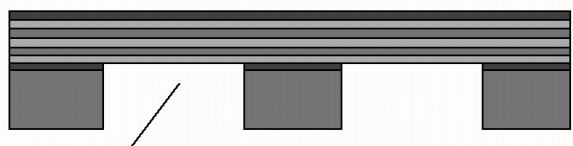

Deep plasma RIE

(b)

Figure 4. Process flow of the device wafer, containing a $2.52 \mu \mathrm{m}$ multilayer diaphragm used for vacuum monitoring.

\section{EXPERIMENTAL RESULTS}

As stated above, the diaphragms are released in a DRIE etcher after the wafers are bonded together. Figure 5(a) shows the photograph of a full bonded wafer pair. Inspection of the wafer shows a yield of more than $95 \%$ of the packaged diaphragms deflecting down across the wafer. Figures $5(b, c)$ show close-ups of the buckled diaphragms over the vacuumsealed cavity. Note that the thin diaphragm bends by a large 
amount and still survives the one atmosphere of pressure difference across it. Figure 6 shows a SEM photograph of the cleaved bond interface, showing the eutectic region. Figure 7 shows a photograph of a device where the cap wafer is forcefully broken and removed, leaving behind silicon in the bond areas. This illustrates a very uniform and strong bond.

The pressure in the sealed cavity can be grossly monitored by monitoring the deflection of the diaphragm as a function of pressure and time. When the bonded wafer is placed inside a vacuum chamber, the diaphragms begin to move up as the chamber is pumped down and the differential pressure across the diaphragm decreases. Several wafers have been bonded and tested in this fashion. The longest wafer has been tested for more than 14 weeks, and the diaphragms are still deflected in ambient pressure. The above technique is not the most accurate to measure and monitor the pressure inside the cavity. To do this, one needs vacuum sensors. We are currently fabricating a set of packages with integrated vacuum sensors that should allow us to directly measure the package pressure down to $1 \mathrm{mT}$ orr.

In addition to bonding cap wafers to flat device wafers without any feedthroughs, we have also bonded the cap wafer to a device wafer with $1.2 \mu \mathrm{m}$ thick feedthroughs. As previously mentioned, the advantage of the eutectic is that it flows over these feedthroughs. The wafers bonded with these feedthroughs also show excellent uniformity and reproducibility.

\section{DISCUSSION}

In order to characterize and understand the wafer boning process, we have also bonded many different wafers with different set of materials and bonding layers. The material sets which were bonded and tested included: $\mathrm{Si} / \mathrm{Ti} / \mathrm{Au}$ to $\mathrm{Au} / \mathrm{Ti} / \mathrm{Si}$; $\mathrm{Si} / \mathrm{Ti} / \mathrm{Au}$ to $\mathrm{Si} ; \mathrm{Si} / \mathrm{Ti} / \mathrm{Au}$ to PolySi/Si; $\mathrm{Si} / \mathrm{Ti} / \mathrm{Au}$ to Oxide/Si; and $\mathrm{Si} / \mathrm{Ti} / \mathrm{Au}$ to Nitirde/Si. Of these wafers, we have found that the bond quality and uniformity between $\mathrm{Au}-\mathrm{Au}, \mathrm{Au}-\mathrm{Si}$, and $\mathrm{Au}-$ PolySi is the best. In particular, it is noted that the bond quality is best when the gold and silicon are supplied from two different wafers, instead of from the same wafer. Test wafers where the $\mathrm{Au}-\mathrm{Si}$ eutectic was bonded to a substrate covered with either oxide or nitride produced very non-uniform and poor bonds. The reasons for this are not exactly known at this point, but the following discussion explains what we believe is occurring in the bonding process.

To help illustrate this, we refer to Figure 8. Let's consider the case of a cap wafer with a gold-silicon eutectic ring being bonded to a silicon oxide surface. The surface of the oxide is generally terminated by $-\mathrm{OH}$ groups. During eutectic bonding, silicon atoms from the cap wafer diffuse through the gold layer, and arrive at the top surface of the oxide, where they either establish a weak chemical bond with $-\mathrm{H}$, or react with $-\mathrm{OH}$ groups to form a $\mathrm{Si}-\mathrm{O}$ bond. This results in the generation of hydrogen, which in turn forms micro- or nano- voids along the bond interface. These are the cause of a weak bond between the eutectic alloy and the oxide.

If LPCVD polysilicon or silicon is used as bonding material on the device wafer, a strong bond is obtained. The polysilicon layer is deposited immediately after the deposition of a LPCVD oxide layer on the device wafer; a strong chemical bond exists between silicon and oxygen atoms along the interface between the oxide and the polysilicon (Fig. 9(a)). When the surface of the polysilicon (or $\mathrm{Si}$ ) is exposed to air and water, a layer of $-\mathrm{OH}$ group forms on the surface (Fig. 9(a)). A very thin layer of oxide is also formed. This very thin native oxide and $-\mathrm{OH}$ layers are consumed by $\mathrm{Si}$ atoms that diffuse through the Au layer and reach the interface, thus forming a strong diffusional bond at the interface of $\mathrm{Au}$ and polysilicon. Although in some regions $\mathrm{Au}$ atoms arrive at the interface between the polysilicon and the oxide, the strong bond between $\mathrm{Si}-\mathrm{Si}$ is not affected (Fig. 9(b)) therefore there still remain a large number of $\mathrm{Si}-\mathrm{Si}$ bonds along the interface. Consequently a very strong and uniform bond establishes across the entire interface. The above mechanisms can also be applied to both nitride and poly/nitide.

We have also studied the effect of the cooling rate on the quality of the eutectic bond. It is well-known that the physical properties of a metal alloy, such as gas diffusivity, depends on the microstructure which depends on such variables as the

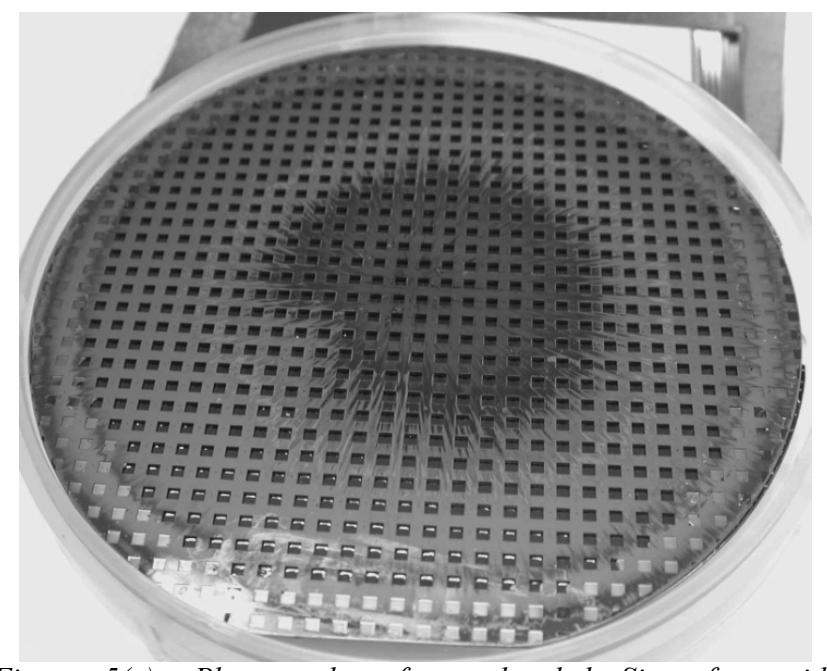

Figure 5(a): Photograph of two bonded Si wafers with cavities covered by thin diaphragms. The yield is $>95 \%$.

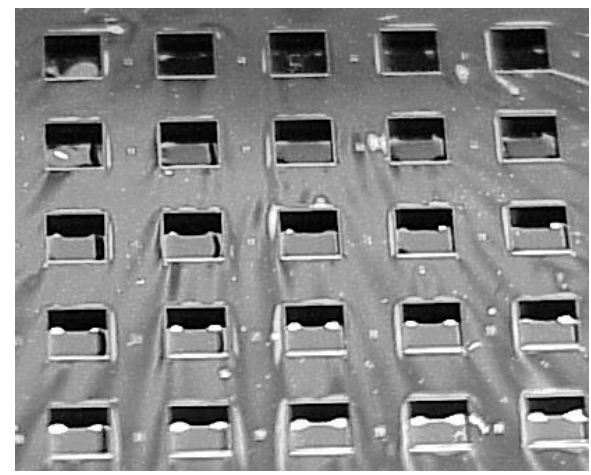

Figure 5(b): Close-up view of some of the vacuum sealed cavities, showing buckled diaphragms.

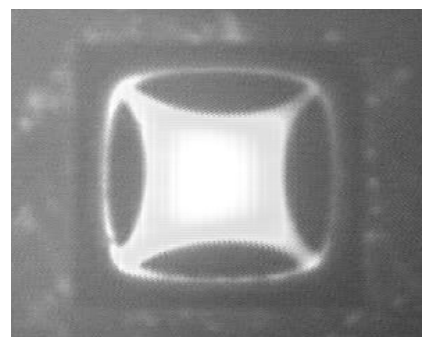

Figure 5(c): Close-up with of a bucled diaphragm covering a sealed cavity. The diaphragm has bucked down by $\sim 28 \mu \mathrm{m}$. 


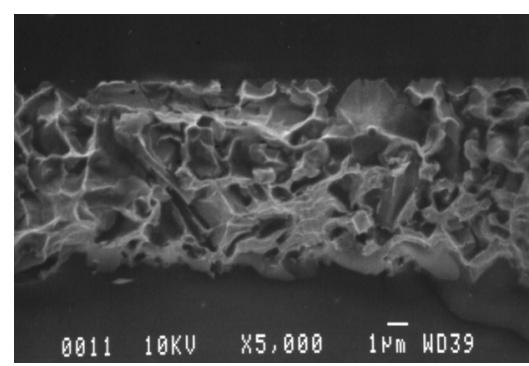

Figure 6. SEM of cross-section of the bond interface.

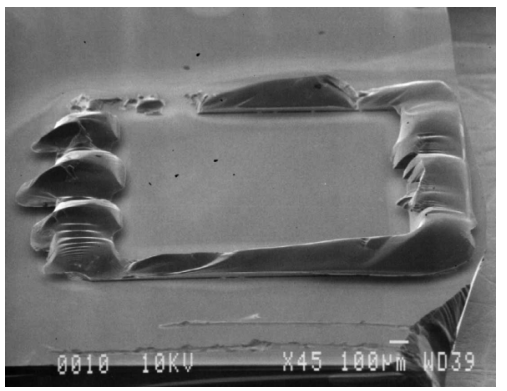

Figure 7. SEM of broken bond region showing excellent uniformity.

alloying elements present, their concentrations, and the heat treatment of the alloy. As shown in Fig. 1, at $>363^{\circ} \mathrm{C}$, the Au$\mathrm{Si}$ alloy is completely liquid (of composition $19 \% \mathrm{Si}$ ). At the eutectic point, the solidification process starts and solids of $\mathrm{Au}$ and $\mathrm{Si}$ begin to form. For packaging applications, one needs a fine (uniform) microstructure in the alloy because smaller grains are more impervious to gas diffusion. Fast cooling (quenching) of the eutectic results in a fine microstrucrture, and is therefore preferred. We have analyzed the microstructure of both quenched and slowly-cooled Au-Si eutectic, and observed that the quenched eutectic has a finer microstructure.

The above proposed Au-Si eutectic bonding experiments indicate that the Au-Si eutectic bond must be conducted: (a) in a vacuum or inert gas ambient to avoid further oxidization at high temperature; (b) LPCVD polysilicon must be deposited immediately after LPCVD oxide deposition to achieve a clean and strong interface between silicon and oxide; (c) some contact force must be applied on the wafers to provide an intimate contact between the bonding materials ( $\mathrm{Au}$ and polysilicon); (d) the bonded wafers should be cooled down as fast as possible around the eutectic temperature; (e) the thickness of the eutectic material should be several microns in order to provide sufficient $\mathrm{Au}-\mathrm{Si}$ eutectic, so when the wafers are pressed together, the eutectic can cover over non-planar surfaces; and (f) the wafers should be properly baked to minimize out-gassing.

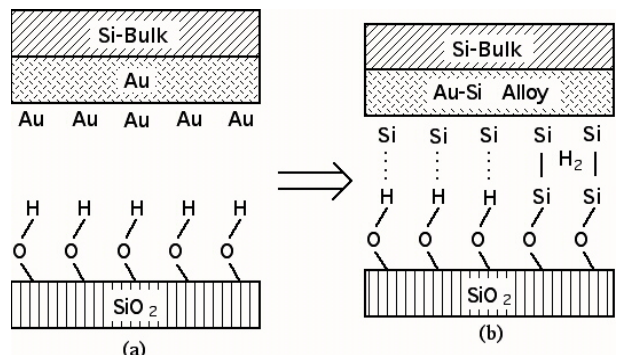

Figure 8. Schematic drawing of atomic configurations at bonding interfaces with LPCVD oxide only.
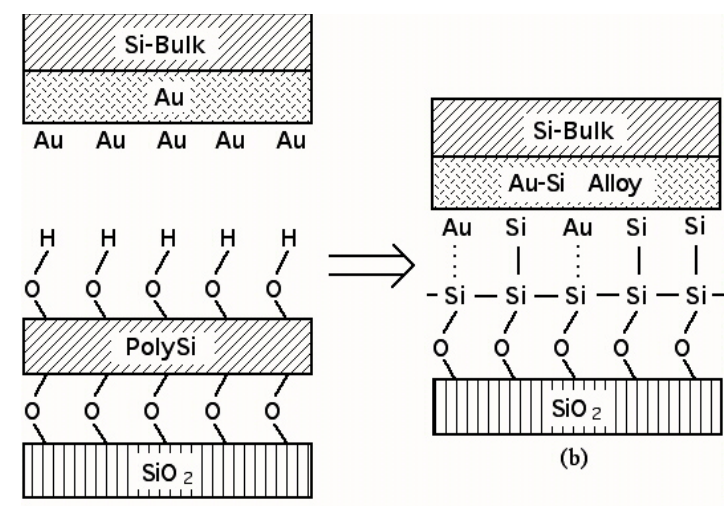

(b)

(a)

Figure 9. Schematic drawing of atomic configurations at bonding interfaces with LPCVD oxide and polysilicon.

\section{CONCLUSIONS}

We have successfully demonstrated a uniform, high-yield, reproducible, gold-silicon eutectic wafer-level bonding technology and its application to MEMS vacuum packaging. Uniform bonding has been achieved across 4" silicon wafers with better than $95 \%$ yield on both flat wafers and wafers with feedthroughs. The best bonds are achieved on wafer pairs where the cap wafer is made of silicon with an electroplated layer of gold, and a device wafer with a polysilicon bond ring deposited over insulated feedthroughs. Vacuum-sealed cavities have been tested by monitoring the deflection of thin flexible diaphragms covering the sealed cavities, and show stable pressure inside the sealed cavity after 14 week.

\section{ACKNOWLEDGEMENTS}

The authors acknowledge the support of Integrated Sensing Inc. (ISSYS) for wafer testing. This research is supported by the Engineering Research Centers Program of the National Science Foundation under Award Number EEC-9986866.

\section{REFERENCES}

1. R.F. Wolffenbuttel, "Low-Temperature Intermediate Au-Si Wafer Bonding: Eutectic or Silicide Bond", Sensors and Actuators, A-62 (1997), pp. 680-686.

2. F. Ohara, et al., "Method for Manufacturing a Semiconductor Acceleration Sensor Device," U.S. Patent 5668033, 1997

3. M .B. Cohn, K. F. Bohringer, J. M. Noworolski, A. Singh, C. G. Keller, K. Y. Goldberg, R. T. Howe, "Microassembly Technologies for MEMS", Proceeding of SPIE, 3512 (1998), pp. $2 \sim 16$.

4. B. Ziaie, J. A. V. Arx, M.R. Dokmeci, and K. Najafi, "A Hermetic Glass-Silicon Micropackage with High-Density OnChip Feedthroughs for Sensors \& Actuators", JMEMS, Vol. 5, No. 3, September (1996), pp. 166-179.4.

5. Y. T. Cheng, W.T. Hsu, Liwei Lin, C.T. Nguyen, and K. Najafi, "Vacuum Packaging Technology Using Localized Aluminum/Silicon-To-Glass Bonding", MEMS 2001, Interlaken, Switzerland, Jan. (2001), pp.18-21.

6. R. K. Shukla and N.P. Mencinger, "A Critical Review of VLSI Die-Attachment in High Reliability Applications", Solid State Technology, July (1985), pp. 67-74. 